\title{
A New Tandem Mass Spectrometer for the Study of Molecular Dissociations
}

\author{
G. $\mathrm{Li}^{*}$ A. Duhr, and H. Wollnik \\ II. Physikalisches Institut der Justus-Liebig-Universität, Giessen, Germany
}

\begin{abstract}
A new tandem mass spectrometer has been developed for the study of molecular fragmentation reactions. The first stage of this tandem mass spectrometer is a double-focusing sector field instrument in the $\mathrm{BE}$ configuration. The second stage is a double-focusing combination of a Wien filter and a sector magnet that allows simultaneous detection of a wide range of masses owing to the approximately equal velocities of the fragment ions. By using a microchannel plate as a collision target, high fragmentation efficiency and high ion transmission were achieved. (J Am Soc Mass Spectrom 1992, 3, 487-492)
\end{abstract}

$\mathrm{T}$ o investigate the molecular structures of large molecules, the tandem mass-spectrometric method has become a useful lool. Here a certain molecular ion is mass-selected by the first stage of a tandem mass spectrometer (MS-I) and then dissociated by using ion-gas or ion-surface collisions. In the second stage (MS-II), the fragment ions can then be mass-analyzed.

Generally, fragmentation of molecular ions can be achieved by two different methods, collision-induced dissociation (CID) [1] or surface-induced dissociation (SID) [2]. In CID, the selected molecular ions are focused into a collision cell in which the molecular ions are activated by collisions with gas atoms or molecules. In this case, the kinetic energy that can be transferred into molecular excitations is limited, usually by the small mass ratio of the collision gas atoms to the molecular ions. In SID, the selected molecular ions are activated by collisions with metal surfaces, in which case a large fraction of the kinetic energy of an ion may be converted into excitation energy.

Decisive factors in such reactions are high fragmentation efficiencies and high collision energies. According to such considerations, the SID method is more advantageous than the CID method thanks to larger collision cross sections and higher energy conversions. In addition, using a surface target does not degrade the residual gas pressure in the vacuum system. The ion transmission in the SID method, however, is usually decreased by the ion-optical configuration of the target because molecular ion beams are typically focused onto the target surface at an incident

\footnotetext{
*This article is a part of the doctoral thesis of $\mathrm{G}$. $\mathrm{Li}$.

Address reprint requests to Herman Wollnik, II. Physikalisches Institut, Justus-Liebig-Universität, Heinrich-Buff-Ring 16, D-6300 Giessen, Germany.
}

angle of about $45^{\circ}$ [2]. A symmetrical extraction optic for the fragment ions is thus difficult to realize.

A new SID method has been developed by Aberth $[3,4]$, in which the inner surfaces of a microchannel plate are used as the target. In this method, the precursor ions collide with the inner channel surfaces at incident angles of about $1-5^{\circ}$ (see Figure 1). This method has two advantages: (1) Small neutralization effects of the molecular ions owing to a high surface resistance of the microchannels and (2) a simple ionoptical design for ion collision and fragment extraction.

A tandem mass spectrometer can be built in various ways [5]. In most cases, a combination of two sector field spectrometers $[6,7]$ or of a radiofrequency (RF) quadrupole and a sector field are used [8].

\section{Second-Stage Mass Spectrometer}

In the tandem mass spectrometer described here, a double-focusing sector field in reverse Nier-Johnson geometry [9] is used as the first stage. The second stage is the combination of a Wien filter [10] with a magnetic sector field as proposed by Matsuda and Wollnik [11].

\section{Ion-optical Considerations}

For molecular fragmentation that releases small energies compared to the kinetic energy of the incoming molecular ion, all fragment ions leave the collision cell or target with approximately the velocity of the incoming molecular ions. Consequently, the kinetic energies of the fragment ions are proportional to their masses. The simplest way to analyze such fragments is to use a single magnetic or electrical sector field. 


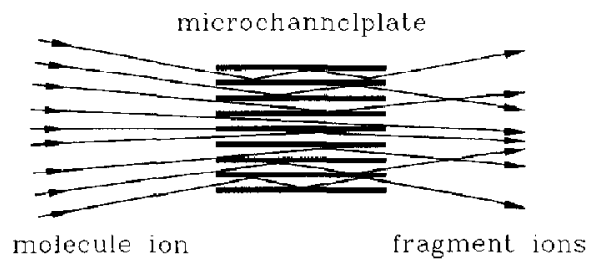

Figure 1. Illustration of the trajectories of molecular ions and their fragments formed by collisions with the inner surfaces of microchannel plates.

The mass-resolving power of such an instrument, however, is limited. A better approach is to use a double-focusing instrument in the BE- or EB-configuration. The use of any electrostatic sector field is especially difficult here because in an electrostatic field the radii of ions of different masses are drastically different. This results from the fact that the mass-to-energy ratio of all fragment ions equals approximately the mass-to-energy ratio of precursor ions because of equal velocities. Thus ions of too low or too high masses are easily lost to the electrodes. Using a Wien filter prevents this from happening. Thus a double-focusing combination of a Wien filter and a sector field magnet (see Figure 2) should be advantageous $[11,12]$.

\section{Ion-optical Properties of a Wien Filter}

$A$ Wien filter consists of a magnetic field $B$ and an electric field $E$ that are perpendicular to each other and to the optical axis. The force $F$ on a reference ion of charge $q$ moving along the optical axis in the Wien filter with velocity $v_{0}$ is as follows (see Figure 3):

$$
F=q\left(E-v_{0} B\right)
$$

This reference ion passes through the Wien filter undeflected if $E$ and $B$ are chosen such that $v_{0}=E / B$ independent of the charge of the ion. Consequently, ions with velocities $v_{0} \pm \Delta v$ are deflected while passing through the Wien filter. Generally [10], we can

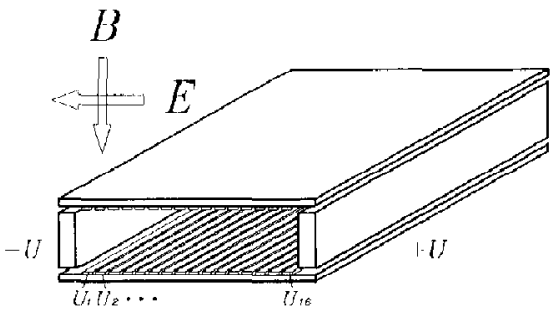

Figure 3. For a Wien filter, the electrostatic field $\vec{E}$ is perpendicular to the magnetic flux density $\vec{B}$. Note the electrodes at potentials $\pm U$ and the potential divider electrodes arranged along the pole faces of the Wien filter magnet.

derive a transfer matrix $\mathbf{W}$ for an ion of velocity $v=v_{0}(1+\Delta)$ :

$$
\begin{aligned}
& \left(\begin{array}{ccc}
(x \mid x) & (x \mid a) & (x \mid \Delta) \\
(a \mid x) & (a \mid a) & (a \mid \Delta) \\
0 & 0 & 1
\end{array}\right) \\
& =\left(\begin{array}{ccc}
\cos (w) & \rho_{b} \sin (w) & \rho_{B}(1-\cos (w)) \\
-\sin (w) / \rho_{B} & \cos (w) & \sin (w) \\
0 & 0 & 1
\end{array}\right)
\end{aligned}
$$

where $w=L_{w} / \rho_{B}$ with $L_{w}$ being the length of the Wien filter and $\rho_{B}$ the deflection radius of ions in the event that the electric field is switched off. From this transfer matrix one reads that the velocity dispersion of the Wien filter is $\rho_{B}\left[1-\cos \left(L_{w} / \rho_{B}\right)\right]$. Combining a sector field magnet characterized by a transfer matrix B with a Wien filter, we find that the velocity dispersions of both elements can compensate for each other. Drift distances $L_{1}$ and $L_{2}$ before the Wien filter and behind the sector field magnet finally cause a transfer matrix for the whole system:

$$
\left(\begin{array}{ccc}
(x \mid x) & (x \mid a) & (x \mid \Delta) \\
(a \mid x) & (a \mid a) & (a \mid \Delta) \\
0 & 0 & 1
\end{array}\right)=\left(\mathbf{L}_{2}\right)(\mathbf{B})(\mathbf{W})\left(\mathbf{L}_{1}\right)
$$

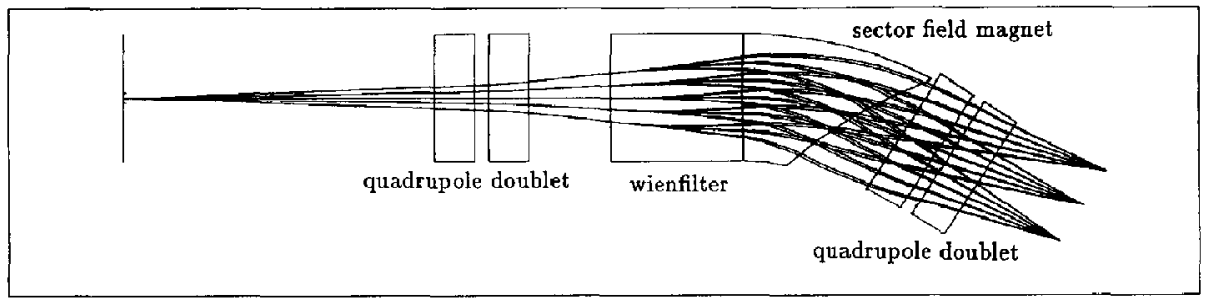

Figure 2. A double-focusing mass spectrometer consisting of a Wien filter and a sector magnet that share the same dipole magnet. Note that before and behind the dipole magnet are placed electric quadrupole doublets that ensure the focusing conditions for variable magnetic and electrostatic dipole fields also and that further allow variation of the mass dispersion. 
For the velocity-focusing condition $(x \mid \Delta)=0$, we find the condition

$$
\rho_{n}\left[\cos \left(\frac{L_{w}}{\rho_{B}}+\Phi_{B}\right)-1\right]=\mathbf{L}_{2} \sin \left(\frac{L_{w}}{\rho_{B}}+\Phi_{B}\right)
$$

where $p_{0}$ is the radius of deflection in the magnetic sector field and $\Phi_{B}$ the corresponding angle of deflection. For the angle-focusing condition $(x \mid a)=0$, we find

$$
\tan \left(\Phi_{B}+\frac{L_{w}}{\rho_{B}}\right)=\frac{\mathbf{L}_{1} \mathbf{L}_{2}-\rho_{0}^{2}}{\rho_{0}\left(\mathbf{L}_{1}+\mathbf{L}_{2}\right)}
$$

Because $\rho_{0}$ and $\rho_{B}$ depend on the ion mass, eq 5 is not fulfilled for ions of different masses. To achieve a good angle focusing over the full mass range, electrostatic quadrupole lenses can be inserted into the system to compensate this dependence [13]. Note here that the focal length $F_{w}$ for a Wien filter and $F_{Q}$ for a quadrupole lens are as follows:

$$
\begin{aligned}
& F_{w}=\frac{\rho_{B}}{\sin \left(L_{w} / \rho_{B}\right)} \\
& F_{Q}=\frac{1}{k \sinh \left(k L_{Q}\right)}
\end{aligned}
$$

where $k=\mathrm{G}_{0}^{-1} \sqrt{2 U_{q} / m v^{2}}$. Here $L_{Q}$ is the length and $G_{0}$ the aperture radius of the quadrupole with $\pm U$ being the potentials on the four electrodes. If the electrode potential is chosen such that $F_{Q}=-F_{w}$, the system becomes mass-independent over a wide mass range.

\section{Nonhomogeneous Electrostatic Field}

The elements $(x \mid \Delta)$ and $(x \mid a)$ of the transfer matrix in eq 3 vanish if eqs 4 and 5 are fulfilled simultaneously (i.e., the system becomes double-focusing to the first order). However, in general the image aberrations of higher orders remain large, thus reducing the resolving power. A correction of the elements of the second order in $a$ of the transfer matrix is very important, especially for the case when ions leave a collision cell over large angles. The element $(x \mid a a)$ can be corrected if a nonhomogeneous electrostatic or magnetic field is used. For technical reasons, we use in our Wien filter mass spectrometer an electrostatic field in which two sets of sixteen electrodes each control this nonhomogeneity $[14,15]$. These electrodes are arranged above and below the deflection plates of the Wien filter placed in the vacuum chamber between the magnet poles and parallel to the optical axis (see Figure 3).
Generally, the potential distribution in a nonhomogeneous electrostatic sector field can be given as

$$
U(x, y, z)=U(x, y, 0)
$$

if the potential along the optical axis is constant, as shown in Figure 3. After expanding $U(x, y)$ in a power series around $(x=y=0)$, we obtain

$$
\begin{aligned}
U(x, y)= & -\chi_{E}\left[a_{0}-\frac{x}{\rho_{E}}-\frac{a_{1}}{2}\left(\frac{x^{2}}{\rho_{E}^{2}}-\frac{y^{2}}{\rho_{E}^{2}}\right)\right. \\
& \left.-\frac{a_{2} x}{3 \rho_{E}}\left(\frac{x^{2}}{\rho_{E}^{2}}-3 \frac{y^{2}}{\rho_{E}^{2}}\right)-\cdots\right]
\end{aligned}
$$

where $\chi_{E}=E \rho_{E}$ is the electrostatic field strength at the optical axis multiplied by the radius of that axis $[11,14]$. Here $a_{0} X_{E}=U(0,0)$ is the potential on the optical axis. Furthermore, $a_{1}$ and $a_{2}$ denote first and second derivatives of the electrostatic potential $U(x, y)$ with respect to the $x$ direction. For $U(0,0)=0$ and a vanishing $d U / d x$ (i.e., for $a_{0}=a_{1}=0$ ), eq 9 simplifies to

$$
U(x, y)=\frac{\chi_{E}}{\rho_{E}} x\left[1+\frac{c_{2}}{6}\left(x^{2}-3 y^{2}\right)+\cdots\right]
$$

where $c_{2}=d / d x\left(1 / \rho_{E}\right)=2 a_{2} / \rho_{E}^{2}$. Here $c_{2}$ must be modified so that $(x \mid a a) \approx\left(x \mid \delta_{K} \delta_{K}\right) \approx 0$, for instance, by using the computer code CIOS [16, 17].

\section{Variable Mass Dispersion}

To study ion-molecular reactions, a simultaneous detection of a wide range of ion masses is often desirable. This can be achieved with a position-sensitive detector. Here the acceptable mass range depends on the angular acceptance of the detector as well as on the mass dispersion $\left(x \mid \delta_{m}\right)$ of the spectrometer.

To change the mass dispersion and thereby vary the mass range of the detector, a variable quadrupole doublet is added after the sector field magnet [10, 13]. Figure 4 shows the intensity distribution of ions of

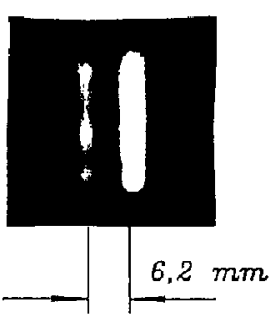

$a$

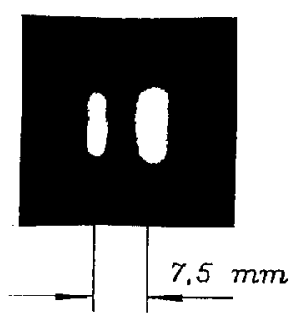

$b$
Figure 4. Observed mass doublets $17(a)$ and $18(b)$ at different settings of the quadrupole doublet behind the Wien filter. Note that the quadrupole doublet before the Wien filter in each case was adjusted to achieve simultaneous angle and energy focusing. 
masses 17 and 18 in the focal plane at different quadrupole potentials. Note here that varying the potential of the quadrupoles changes the mass dispersion $\left(x \mid \delta_{m}\right)$ but not the mass resolving power $\left(x \mid \delta_{m}\right) /(x \mid x)$ because the matrix element $(x \mid x)$ changes equally.

\section{Experimental}

The experimental setup of our tandem mass spectrometer is shown in Figure 5. For these experiments, ions were formed exclusively by electron ionization and fragmented at the microchannel plate collision cell indicated in Figure 1.

The collision chamber is designed as a complete device for molecular ion detection and fragmentation. A double microchannel plate detector in chevron arrangement behind a narrow slit and the collision microchannel plate are mounted together in a movable arrangement.

The collision microchannel plate was placed together with some deceleration and acceleration plates in the focal planc between the double-focusing mass spectrometer and the double-focusing Wien filter system. The front plane of the microchannel plate-a Galileo MCP-25 (Galileo Optics, Sturbridge, MA) with channel diameters of $10 \mu \mathrm{m}$, thickness of $0.43 \mathrm{~mm}$, bias angle of $4.6^{\circ}$, and an open area ratio of $63 \%$-was connected to a variable-voltage source that determined the collision energy. An acceleration voltage was applied to the back plane of the microchannel plate during all experiments, providing for a high transmission of fragment ions. Some deflection plates were also used to alter the direction of motion of the fragment ions leaving the collision cell.

The properties of the double-focusing Wien filter were tested by using a separate ion source operated with $\mathrm{Xe}^{+}$(see Figure 6). The ion energy here was 2 $\mathrm{keV}$, and the achieved mass resolving power $m / \Delta m$ $\approx 2600$, which seemed adequate for the intended application. The performance of the full tandem massspectrometric system is illustrated in Figure 7 . For this illustration, singly charged pyrene ions of $m / z=202$ were used. After mass selection by the first stage (i.e., the double-focusing BE-mass spectrometer operated at an acceleration potential of $3 \mathrm{kV}$ ), these ions were decelerated to $1,1.3$, and $1.6 \mathrm{keV}$, respectively, before shooting them into the microchannel plate for fragmentation. The fragment ions were then massanalyzed by the second-stage double-focusing Wien filter system.

As can clearly be seen in Figure 7, the pyrene ions arc increasingly fragmented at higher kinetic energies, although the mass resolving power for the frag-

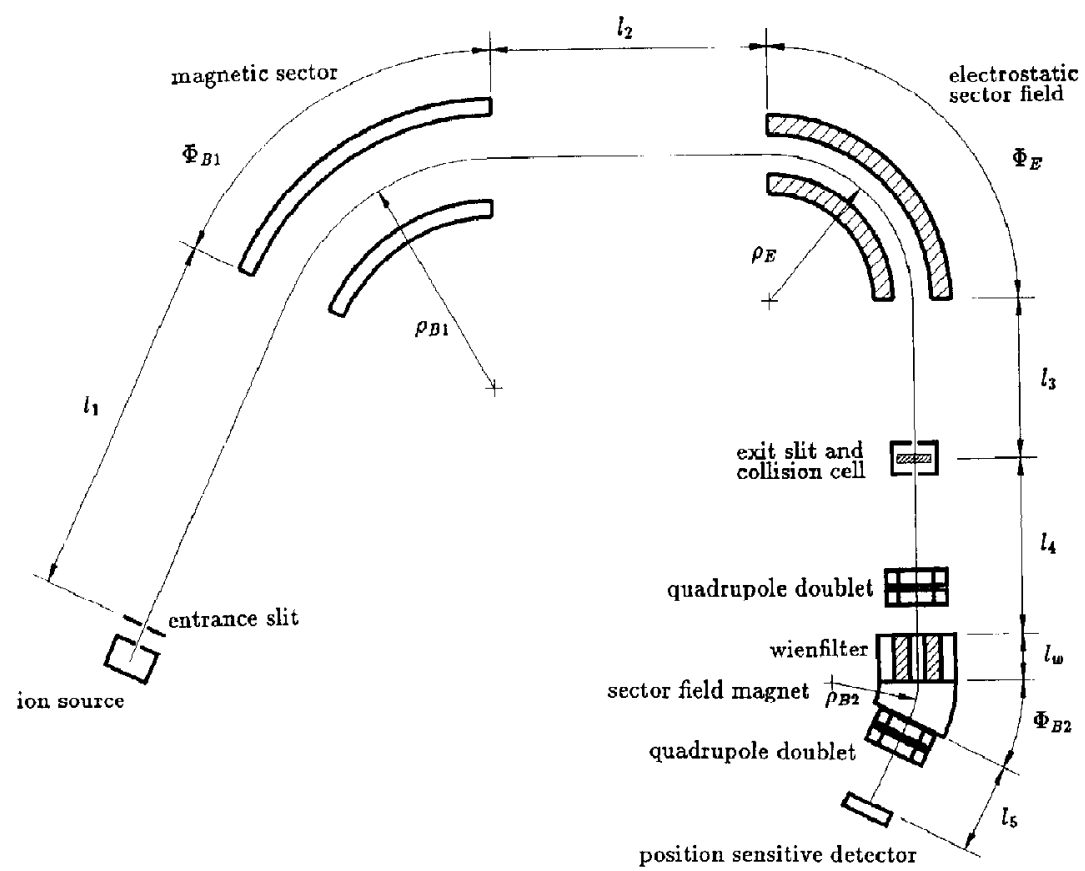

Figure 5. Arrangement of the tandem mass-spectrometric system. The parameters of the frst stage are $l_{1}=1.120 \mathrm{~m}$ and $l_{2}=0.871 \mathrm{~m}$, with the homogeneous magnetic sector field characterized by $\Phi_{B}=65^{\circ}$ and $\rho_{B}=0.70 \mathrm{~m}$ and the electrostatic toroid condenser by $\Phi_{E}=90^{\circ}$ and $\rho_{E}=0.448$ $\mathrm{m}$. The parameters of the second stage are $l_{3}=0.487 \mathrm{~m}, l_{4}=0.541 \mathrm{~m}$, and $l_{5}=0.285 \mathrm{~m}$, with the Wien filter characterized by $L_{w}=0.146 \mathrm{~m}$ and $B=1.192 \mathrm{~T}$ and the sector magnet by $\Phi_{B}=26.65^{\circ}$ and $\rho_{B}=0.27 \mathrm{~m}$. 
132

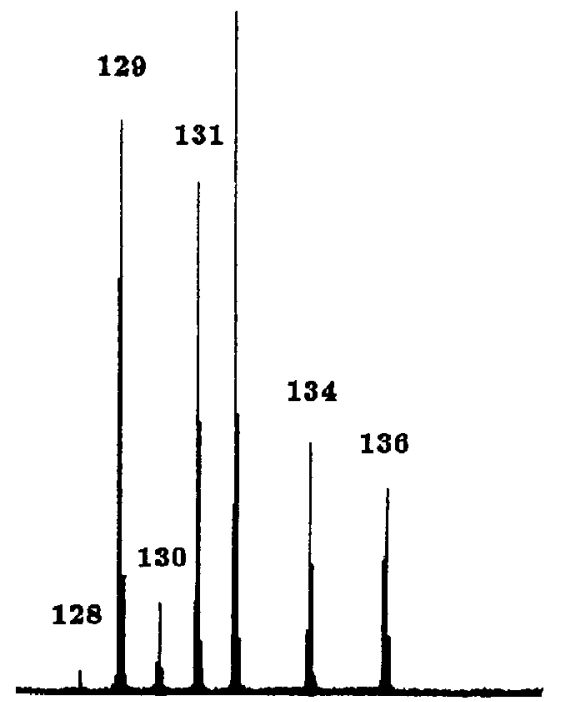

Figure 6. The achieved mass resolving power $m / \Delta m \approx 2600$ of the Wien filter is illustrated for $\mathrm{Xe}^{+}$ions of $2-\mathrm{keV}$ energy formed in a separate ion source. The spectrum was recorded on a personal computer using a single-point detector.

mented ions was only about 200 (considerably lower than the mass resolving power observed for the separately formed $\mathrm{Xe}^{+}$ions). This is a result of the acceleration voltage applied to the fragmentation microchannel plate. In our experiment, this acceleration caused a velocity spread of $3-10 \%$ in the secondary ion beam, which causes a reduced mass resolving power. How-

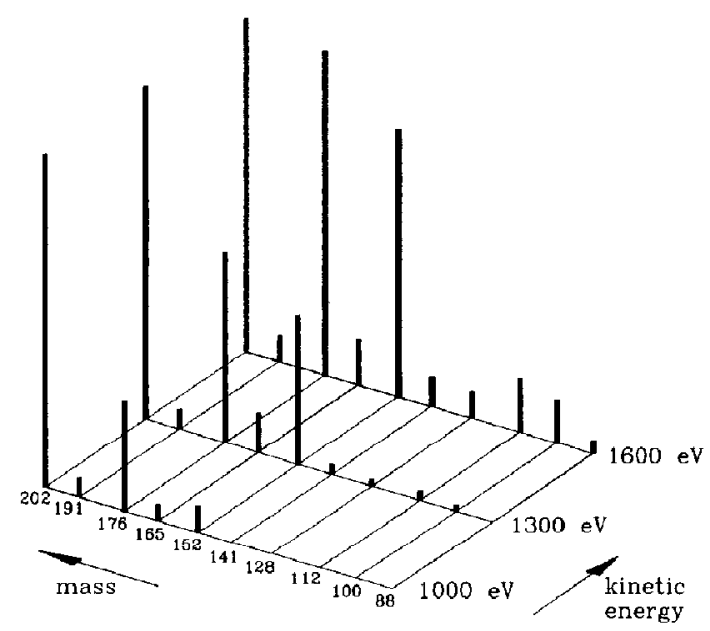

Figure 7. The mass distribution of fragment ions of $m / z 202$ (pyrene). The fragmentation occurred at the inner surfaces of a microchannel plate at energies of $1,1.3$, and $1.6 \mathrm{keV}$ kinetic energy. Note that with increasing ion energy, the smaller fragment ions increase in number considerably.

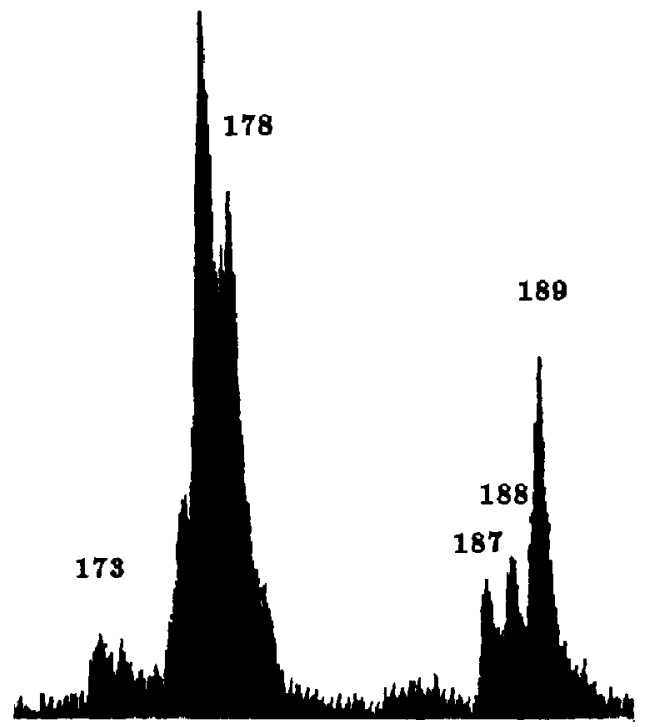

Figure 8. The mass distribution of fragment ions around $m / z$ 178 formed by fragmenting pyrene at $1300 \mathrm{eV}$ in the microchannel plates. This spectrum was recorded with a focal-plane position-sensitive detector.

ever, this voltage increased the sensitivity considerably.

Differing from the energy gained in the channel plate, the additional velocity gained by the fragmented ions in the postacceleration field can be taken into consideration by changing the electric field strength in the Wien filter to meet the velocity of a reference mass ion. However, this slightly reduces the transmission of ions of different masses recorded simultaneously in a position-sensitive detector.

\section{Conclusion}

A novel double-focusing fragment mass analyzer consists of a combination of a Wien filter and a magnetic sector field. For this system, surface-induced dissociation is used, the surface consisting of the inner surfaces of microchannel plates. The system has distinct advantages for high energy collisions over ones that consist of two BE or EB double-focusing sector arrangements. The use of fragment ions of different kinetic energies than the precursor ion is shown to be advantageous.

\section{Acknowledgments}

For many fruitful discussions we are grateful to Prof. $H$. Matsuda. For financial support we are thankful to the Deutsche Forschungsgemeinschaft (DFG), and we are likewise thankful to Finnigan MAT, Bremen, for letting us use one of their magnets. 


\section{References}

1. McLafferty, F. W. Acc. Chem. Res. 1980, 13, 30.

2. Cooks, R. G.; Ast, T.; Mabud, M. A. Int. I. Mass Spectrom. Ion Phys. 1990, 100, 209.

3. Aberth, W. Proceedings of the $36 \mathrm{th}$ ASMS Conference on Mass Spectrometry and Allied Topics, 1988, 73.

4. Aberth, W. Anal. Chem. 1990, 62, 609

5. Boerboom, A. J. H. Rapid Contmun. Mass Spectrom. 1990, 4, 385.

6. McLafferty, F. W.; Todd, P. J.; McGilvery, D. C.; Baldwin, M. A. I. Am. Chem. Soc. 1980, 102, 3360.

7. Gross, M. L., et al. Int. I. Mass Spectrom. Ion Proc. 1982, 42, 243.

8. Schoen, A. E.; Amy, J. W.; Ciupek, J. D.; Cooks, R. G.; Dobberstein, P.; Jung, G. Int. I. Mass Spectrom. Ion Proc. 1985, 65, 125.
9. Wendel, W. Doctoral thesis, Justus-Liebig-Universität, Giessen, Germany, 1986 (unpublished).

10. Aberth, W.; Wollnik, H. Mass Spectrom. Rev. 1990, 9, 383.

11. Matsuda, H.; Wollnik, H. Int. J. Mass Spectrom. Ion Proc. 1988, 86, 53.

12. Li, G.; Ningel, K. P.; Wollnik, H. Verh. DPG 1989, 24, MS 3.5.

13. Wendel, W. Thesis, Justus-Liebig-Universität, Giessen, Germany, 1981 (unpublished).

14. Wollnik, H. Optics of Charged Particles; Academic Press; Orlando, 1987.

15. Wendel, W.; Michener, P.; Wollnik, H. Int. J. Mass Spectrom. Ion Proc. 1988, 90, 131.

16. Wollnik, H.; Brezina, J.; Berz, M.; Wendel, W. Proc. AMCO-7, GSI-Rep. THD-26 1984, 679.

17. Wollnik, H.; Brezina, J.: Berz, M. Nud. Instrum. Meth. 1987, 258A, 408, 